\title{
The influence of rigid gas permeable lens wear on the concentrations of dinucleotides in tears and the effect on dry eye signs and symptoms in keratoconus
}

\author{
Gonzalo Carracedo, ${ }^{\mathrm{a}, \mathrm{c}}$ * José Manuel González-Méijome, ${ }^{\mathrm{b}}$ Alba Martín-Gil,, Jesús Carballo, ${ }^{\mathrm{a}}$ Jesús Pintor ${ }^{\mathrm{c}}$ \\ a Department of Optics II (Optometry and Vision), Faculty of Optic and Optometry, Universidad Complutense de Madrid, Madrid, Spain \\ ${ }^{\mathrm{b}}$ Clinical \& Experimental Optometry Research Lab, Center of Physics (Optometry), School of Sciences, University of Minho, Braga, Portugal \\ ${ }^{c}$ Department of Biochemistry and Molecular Biology IV, Faculty of Optic and Optometry, Universidad Complutense de Madrid, Madrid, Spain
}

\section{A R T I C L E I N F O}

\section{Article history:}

Received 3 January 2016

Received in revised form 19 April 2016

Accepted 26 April 2016

Available online $\mathrm{xxx}$

Keywords:

GP lenses

Keratoconus

Nucleotides

Dry eye

\section{A B S T R A C T}

\section{Purpose}

To evaluate the signs and symptoms of dry eye and dinucleotide secretion in tears of keratoconus patients (KC) and the potential effect of rigid gas permeable (RGP) contact lens wear.

\section{Methods}

Twenty-three KC patients and forty control subjects were enrolled in this study. Signs of dry eye including tear volume, tear stability and corneal staining along with symptoms were assessed using the McMonnies questionnaire. Tears were collected using Schirmer strips, and dinucleotide concentrations in collected tears measured using high pressure liquid chromatography. Values obtained in $\mathrm{KC}$ and controls were compared. The effect of contact lens wear in $\mathrm{KC}$ was also assessed.

Results

KC eyes showed a significantly lower tear volume compared to controls, shorter tear break up time (TBUT), higher corneal staining and higher McMonnies dry eye questionnaire scores $(\mathrm{p}<0.05)$. When compared with non-wearers, KC contact lens wearers showed significantly higher symptoms, lower Schirmer and TBUT values $(\mathrm{p}<0.05)$. Concentration of $\mathrm{Ap}_{4} \mathrm{~A}(0.695 \pm 0.304 \mu \mathrm{M}$ vs. $0.185 \pm 0.178 \mu \mathrm{M})$ and $\mathrm{Ap}_{5} \mathrm{~A}(0.132 \pm 0.128 \mu \mathrm{M}$ vs. $0.045 \pm 0.036 \mu \mathrm{M})$ were higher in $\mathrm{KC}$ compared to controls $(\mathrm{p}<0.001)$ and only $\mathrm{Ap}_{4} \mathrm{~A}$ was statistically higher in RGP wearers compared to non-wearers $(0.794 \pm 0.478 \mu \mathrm{M}$ vs. $0.417 \pm 0.313 \mu \mathrm{M})(\mathrm{p}<0.05)$

\section{Conclusion}

Signs and symptoms of dry eye as well as concentrations of $\mathrm{Ap}_{4} \mathrm{~A}$ and $\mathrm{Ap}_{5} \mathrm{~A}$ were markedly increased in $\mathrm{KC}$ patients compared to controls. Moreover, $\mathrm{Ap}_{4} \mathrm{~A}$ and symptoms of dry eye were statistically higher in RGP wearers compared to non-wearers. This seems to indicate that factors such as RGP contact lens wear might exacerbate the clinical condition of dry eye.

(C) 2016 Published by Elsevier Ltd.

\section{Introduction}

Keratoconus $(\mathrm{KC})$ is an ocular pathology causing a protrusion of the cornea. This pathology is bilateral, asymmetric and progressive $[1,2]$ and affects about $2 \%$ of the population worldwide [2]. The cause of $\mathrm{KC}$ is not well understood, but it seems to be multifactorial. Factors such as an exposure to ultraviolet light, allergy and genetic mechanisms are the predominant reasons triggering the disease [3-5]. Other factors described in the literature as risk factors for the progression of $\mathrm{KC}$ include eye rubbing, with half of the patients reporting intense eye rubbing [6]. The need these patients feel to rub their eyes is due to ocular discomfort symptoms including itching, which might be closely related to eye dryness [7].

Only a very small number of studies have investigated the link between $\mathrm{KC}$ and dry eye [8-10]. In these studies, the results show a lower tear break time (BUT) in KC patients and a marked presence of corneal staining. In addition, patients with $\mathrm{KC}$ have a higher concen-

* Corresponding author at: Department of Optics II (Optometry and Vision), Faculty of Optic and Optometry, Universidad Complutense de Madrid, Madrid, Spain.

Email address: jgcarrac@ucm.es (G. Carracedo) tration of pro-inflammatory molecules such as interleukins and metalloproteinases compared to healthy subjects, this being even more elevated in patients using RGP contact lenses [11-13]. Inflammation is one of the most important signs described in the definition of dry eye given by the DEWS 2007 [14]. All these findings suggest a possible interaction between $\mathrm{KC}$ and dry eye.

In recent years, the presence of diadenosine polyphosphates in human tears and in aqueous humor has been shown $[15,16]$. These molecules can increase tear secretion when instilled topically [17]. Moreover, these substances can increase the production of lysozyme in tears, contributing to ocular surface defense [18]. Additionally, some of these molecules accelerate the rate of corneal wound healing (diadenosine tetraphosphate, $\mathrm{Ap}_{4} \mathrm{~A}$ ) while others inhibit this process (diadenosine pentaphosphate, $\mathrm{Ap}_{5} \mathrm{~A}$ ) [19]. These molecules have been proposed as objective biomarkers of dry eye, since their presence is significantly increased in patients with this disease [20,21]. In a recent study, it has been found that $\mathrm{Ap}_{4} \mathrm{~A}$ tear concentrations were higher in $\mathrm{KC}$ patients than healthy subjects. All patients with $\mathrm{KC}$ were RGP wearers [10]. A higher $\mathrm{Ap}_{4} \mathrm{~A}$ concentration in healthy RGP wearers has also been shown to be related [22].

The release of diadenosine polyphosphates to the tears is caused by shear stress. Corneal epithelial cells release these molecules as a 
consequence of the lid contact during the blinking process [23]. The CLEK study found an increase of about $25 \%$ of corneal scarring in $\mathrm{KC}$ patients wearing RGP contact lenses compared to non-users, possibly partly due to the abrasion caused by the contact lens to the corneal surface [24]. We hypothesized that patients with KC who wear RGP contact lenses have higher tear concentrations of diadenosine polyphosphates.

To the best of our knowledge, there is currently no study that evaluates the influence of RGP lenses on tears and ocular surface physiology in patients suffering from KC. Therefore, the aim of this study is to examine the signs, symptoms and adenosine polyphosphate tear concentrations in patients with and without $\mathrm{KC}$ as well as the influence of RGP contact lens wear on these parameters in $\mathrm{KC}$.

\section{Methods}

\subsection{Patients}

Twenty-three patients (13 males and 10 females) with ages ranging from 20 to 59 (average $34.75 \pm 6.12$ years) with $\mathrm{KC}$ and forty healthy subjects ( 24 males and 16 females) with ages ranging from 20 to 61 $(29.02 \pm 6.92$ years $)$ were recruited at the Clinical \& Experimental Optometry Research Lab (University of Minho, Braga, Portugal) and at the Optometric Clinic of the Faculty of Optic and Optometry (University of Complutense, Madrid, Spain). All patients with KC were diagnosed by an external ophthalmologist and recommended to the research. The study was conducted in compliance with good clinical practice guidelines, institutional review board regulations, informed consent regulations, and the tenets of the Declaration of Helsinki (WMA, 2008) [25]. The protocol of the study was approved by the institutional review board of the University Complutense of Madrid. All the subjects enrolled in the study were adults over 18 years of age, who were able to give informed consent. After the purpose of the study was explained and all doubts clarified to the participants, a consent form was presented and signed by both the patient and the researcher.

KC patients should have been wearing spectacles or aspheric RGP corneal design at least during the last year to be part of the study. $\mathrm{Pa}$ tients with soft contact lenses, hybrid or scleral or previous surgery were excluded. In order to study the influence of RGP lens wearing on the ocular surface integrity, the $\mathrm{KC}$ group was divided into users and non-users of RGP lenses. Control group subjects had all worn soft contact lenses on a monthly wear daily basis during the previous 2 years, except eight subjects who had never worn contact lenses at all. Additional demographic characteristics of the population are shown in Table 1.

Table 1

Demographic characteristics of participants in the study.

\begin{tabular}{|c|c|c|c|c|}
\hline \multirow[t]{2}{*}{ Parameter } & \multirow[t]{2}{*}{ Control } & \multicolumn{3}{|l|}{ Keratoconus } \\
\hline & & Total & $\begin{array}{l}\text { No RGP } \\
\text { wear }\end{array}$ & RGP wear \\
\hline Patients & 40 & $23>-a$ & 11 & 12 \\
\hline $\begin{array}{l}\text { Mean age } \\
\text { (years) } \pm \mathrm{SD}\end{array}$ & $29.02 \pm 6.92$ & $34.75 \pm 6.12$ & $35.96 \pm 4.82$ & $33.11 \pm 3.16$ \\
\hline Age range (years) & {$[20,61]$} & {$[18,59]$} & {$[23,59]$} & {$[18,44]$} \\
\hline $\begin{array}{l}\text { Gender (male/ } \\
\text { female) }\end{array}$ & {$[24,16]$} & {$[13,10]$} & {$[7,4]$} & {$[6,6]$} \\
\hline \multicolumn{5}{|c|}{ Mean keratometry (D) } \\
\hline Flat & $43.87 \pm 2.53$ & $49.46 \pm 6.32$ & $46.97 \pm 3.35$ & $51.97 \pm 5.62$ \\
\hline Steep & $45.31 \pm 1.39$ & $56.35 \pm 8.76$ & $53.25 \pm 6.53$ & $60.81 \pm 6.79$ \\
\hline
\end{tabular}

\subsection{Trials}

Tests of tear volume, tear stability, corneal staining and recording of subjective symptoms including dryness and discomfort using the McMonnies questionnaire were carried out at the beginning of the day before contact lens wear [26,27].

Tear secretion was measured using the Schirmer I test (without anaesthesia). The tear collection was always performed following the Van Bijsterveld criteria [28]. The Schirmer strip (Tear Flo, HUB pharmaceuticals, USA) was placed on the temporal tarsal conjunctiva of the lower lid for $5 \mathrm{~min}$ with the eyes closed. The volume of tears, as millimetres of moistened strip, were recorded and the Schirmer strips were placed in Eppendorf tubes containing $500 \mu \mathrm{L}$ of Ultrapure water, then the samples were frozen at $-80{ }^{\circ} \mathrm{C}$ until the high pressure liquid chromatography (HPLC) analysis was performed [20].

To avoid contaminating the strip with fluorescein, fluorescein was applied five minutes after the Schirmer I test to evaluate tear break up time (TBUT) and corneal staining. In order to warrant repeatability of the staining procedure, a solution was prepared using a $10 \%$ concentration of sodium fluorescein diluted in saline $(\mathrm{NaCl} 0.9 \%)$. For each application, a micropipette with $5 \mu \mathrm{l}$ of diluted fluorescein solution was applied in the inferior conjunctival sac and $20 \mathrm{~s}$ later TBUT was analyzed through a slit lamp at 16x, using a chronograph to record the time to break after the patient was asked to blink twice and then keep their eyes open. The cornea was divided into five areas to record the grade of staining and, as proposed by the Report of the National Eye Institute and Industry-Sponsored Dry Eye Workshop [29], Cornea and Contact Lens Research Unit (CCLRU) grading scales were used [30].

\subsection{Tear preparation and HPLC analysis}

After thawing, the samples were strongly vortexed for $5 \mathrm{~min}$. The strips were carefully rinsed and the liquid in the Eppendorf tube was heated in a $100{ }^{\circ} \mathrm{C}$ bath for 20 min to precipitate proteins. In order to pellet the proteins, the tubes were centrifuged at $4000 \mathrm{rpm}$ for $30 \mathrm{~min}$. Diadenosine polyphosphates are not degraded by this treatment as previously demonstrated [31]. Supernatants were chromatographed through SEP-PAK Accell QMA cartridges [32]. Briefly, $250 \mu \mathrm{L}$ of the supernatant was passed through the cartridges that were previously mixed with $3 \mathrm{~mL}$ of ultrapure water. The elution of the nucleotides and dinucleotides was performed by applying $1 \mathrm{~mL}$ of a solution containing $0.2 \mathrm{M} \mathrm{KCl}, 0.1 \mathrm{M} \mathrm{HCl}$. Prior to injection into the HPLC, samples were neutralised with $\mathrm{KOH}$. These eluents were injected at a volume of $10-100 \mu \mathrm{L}$.

Determination and quantification of diadenosine polyphosphates were performed by HPLC. The chromatographic system consisted of a Waters 1515 isocratic HPLC pump, a 2487 dual absorbance detector and a Reodyne injector, all managed by the Breeze software from Waters. The column was a Novapack C18 $(15 \mathrm{~cm}$ length, $0.4 \mathrm{~cm}$ diameter) from Waters. The system was equilibrated overnight with the following mobile phase: $0.1 \mathrm{M} \mathrm{KH}_{2} \mathrm{PO}_{4}, 2 \mathrm{mM}$ tetrabutyl ammonium, $17 \%$ acetonitrile, $\mathrm{pH} 7.5$ [19]. Detection was monitored at $260 \mathrm{~nm}$ wavelength. All the peaks identified as putative dinucleotides were taken for phosphodiesterase treatment. Phosphodiesterase from $\mathrm{Cro}-$ talus adamanteus, from Sigma (St. Louis, Mo., USA; EC 3.1.4.1.) at a concentration of $1.0 \mathrm{U} / \mathrm{mL}$ was incubated at room temperature for $30 \mathrm{~min}$ with the corresponding putative dinucleotide. The digested products were analysed by HPLC. Peaks were transformed into concentrations by means of external standards of known concentrations of diadenosine polyphosphates. 


\subsection{Statistical analysis}

Data were analysed by statistical package SPSS version 15.0 for Windows (SPSS, Inc., Chicago, IL). The values analyzed are the means $\pm \mathrm{SD}$ of the experiments performed. For statistical analysis, one eye per subject was selected randomly. Normality of samples was analyzed using the Shapiro-Wilk test. Sample size calculations were performed with statistical software (Granmo 6.0; Institut Municipal d'Investigacioń Medica, Barcelona, Spain). With an accepted twosided statistical significance threshold of 0.05 and a risk of 0.20 , for a standard deviation of 0.16 units for the diadenosine tetraphosphate concentration and in order to detect a difference of 0.2 units or more, 13 subjects were needed to find statistically significant differences. Parametric tests were used to compare the studied groups. Differences between the control group and the $\mathrm{KC}$ group were estimated by the Student- $t$ test for independent samples. Due to small sample sizes of KC subgroups, the Mann-Whitney $U$ test was used to compare values between them. $\mathrm{p}<0.05$ was considered statistically significant.

\section{Results}

Of all the $\mathrm{KC}$ subjects enrolled in the study, 16 patients were of grade III, 5 patients of grade IV and 2 patients of grade $\mathrm{V}$, according to the $\mathrm{KC}$ severity score grading scale ( 9 grade III, 1 grade IV and 1 grade $\mathrm{V}$ were included in non RGP wearers and 7 grade III, 4 grade IV and 1 grade $\mathrm{V}$ were included in RGP wearers) [33]. However, no data were recorded about when the patients were diagnosed of KC. Considering that no correlation between grades and any test performed was found, all the eyes were pooled together for further analysis. Moreover, no differences were found between soft contact lens wearers and non-lens wearers in the control group and therefore the control groups were not divided for additional analysis.

Results of the Schirmer test, TBUT, corneal staining, McMonnies score and concentrations of Ap4A and Ap5A are summarized in Table 2. When comparing the control group and the $\mathrm{KC}$ group, the Schirmer test indicated that the control group showed higher tear volume than the KC group $(\mathrm{p}=0.03)$. TBUT was statistically shorter in the $\mathrm{KC}$ group than the control group $(\mathrm{p}<0.001)$. Corneal staining scores were 3 fold higher in the KC patients than in healthy subjects $(\mathrm{p}<0.001)$. Also, the McMonnies questionnaire showed that the $\mathrm{KC}$ subjects had statistically significant higher symptoms scores than the control group $(\mathrm{p}<0.001)$. Among the symptoms more frequently reported by $\mathrm{KC}$ patients were: dryness (59\%) and scratchiness (36\%). Also, they more frequently reported to have uncomfortable eyes in smoke and/or airconditioning conditions. Regarding $\mathrm{Ap}_{4} \mathrm{~A}$ and $\mathrm{Ap}_{5} \mathrm{~A}$ concentration, $\mathrm{KC}$ patients showed higher concentration than control group, this being statistically significant in both dinucleotides $(\mathrm{p}<0.001)$.

Table 2

Comparison between control group and keratoconus group. ${ }^{*} \mathrm{p}$ value $<0.05$. Student- $t$ test for independent samples. For details see Section 2.

\begin{tabular}{lccc}
\hline Test & Control & Keratoconus & P value \\
\hline $\mathrm{Ap}_{4} \mathrm{~A}(\mu \mathrm{M})$ mean $(\mathrm{SD})$ & $0.185 \pm 0.178$ & $0.695 \pm 0.304$ & $<0.001^{*}$ \\
$\mathrm{Ap}_{5} \mathrm{~A}(\mu \mathrm{M})$ mean $(\mathrm{SD})$ & $0.045 \pm 0.036$ & $0.132 \pm 0.128$ & $<0.001^{*}$ \\
Schirmer $(\mathrm{mm})$ mean $(\mathrm{SD})$ & $19.87 \pm 5.71$ & $16.75 \pm 7.65$ & $0.031^{*}$ \\
TBUT $(\mathrm{s}$.$) mean (\mathrm{SD})$ & $12.13 \pm 1.85$ & $6.56 \pm 2.88$ & $<0.001^{*}$ \\
Corneal staining mean $(\mathrm{SD})$ & $0.82 \pm 0.51$ & $2.81 \pm 0.76$ & $<0.001^{*}$ \\
McMonnies score mean(SD) & $7.01 \pm 3.15$ & $10.87 \pm 3.68$ & $<0.001^{*}$ \\
\hline
\end{tabular}

When the KC group was divided into RGP wearers and non-RGP wearers, statistical differences were found in the Schirmer test, TBUT, the McMonnies test and $\mathrm{Ap}_{4} \mathrm{~A}$ concentration $(\mathrm{p}<0.05)$, but not in corneal staining scores and $\mathrm{Ap}_{5} \mathrm{~A}$ concentration $(\mathrm{p}>0.05$, Table 3$)$.

RGP wearers showed less tear volume than the KC non-RGP wearers. Moreover, the tear stability was shorter and symptoms were higher in RGP wearers than spectacle wearers. $\mathrm{Ap}_{4} \mathrm{~A}$ concentration was higher in RGP wearers compared with non-wearers. Fig. 1 summarizes the main outcomes of this study.

Table 3

Comparison between wearers and non-wearers of GP lenses in keratoconus group. ${ }^{*} \mathrm{p}$ value $<0.05$. Mann-Whitney $U$ test. For details see Section 2 .

\begin{tabular}{lccl}
\hline Test & No RGP & RGP wearers & P value \\
\hline $\mathrm{Ap}_{4} \mathrm{~A}(\mu \mathrm{M})$ mean $(\mathrm{SD})$ & $0.417 \pm 0.313$ & $0.794 \pm 0.478$ & $0.041^{*}$ \\
$\mathrm{Ap}_{5} \mathrm{~A}(\mu \mathrm{M})$ mean $(\mathrm{SD})$ & $0.093 \pm 0.086$ & $0.217 \pm 0.176$ & 0.064 \\
Schirmer $(\mathrm{mm})$ mean $(\mathrm{SD})$ & $20.05 \pm 7.45$ & $13.45 \pm 5.89$ & $0.044^{*}$ \\
TBUT (s.) mean (SD) & $8.11 \pm 1.85$ & $5.83 \pm 1.23$ & $0.002^{*}$ \\
Corneal staining mean (SD) & $2.42 \pm 0.46$ & $2.59 \pm 0.29$ & 0.287 \\
McMonnies score mean(SD) & $7.93 \pm 3.56$ & $12.45 \pm 3.32$ & $0.005^{*}$ \\
\hline
\end{tabular}
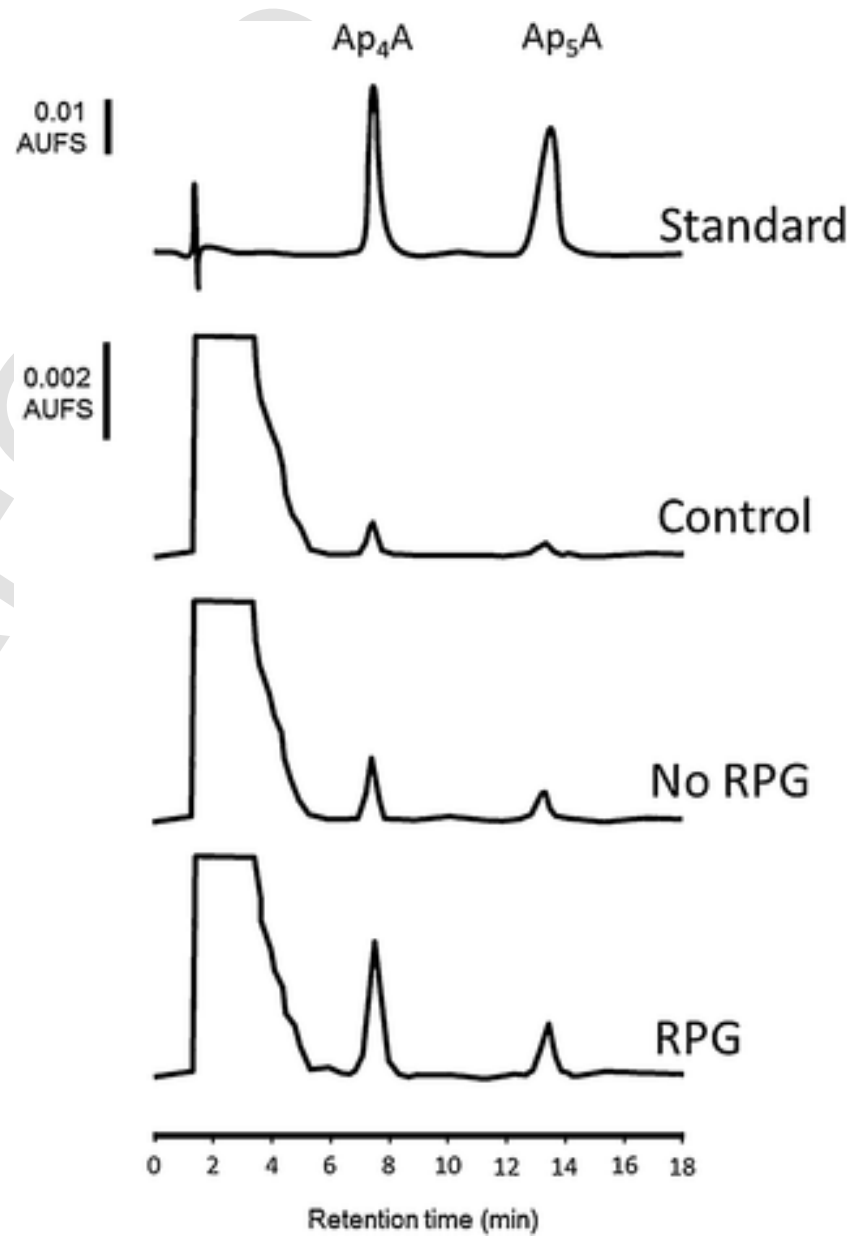

Fig. 1. Diadenosine polyphosphate detection in tears of KC patients. Chromatographic analysis of samples from $\mathrm{KC}$ patients showing the presence of two peaks identified as $\mathrm{Ap}_{4} \mathrm{~A}$ and $\mathrm{Ap}_{5} \mathrm{~A}$ when compared to a commercial standard (upper record). A control record (representing a healthy individual) can be compared with representative traces of KC patients grouped, according to methods, in RGP wearers and non-RGP wearers. Note that standards scale bar is different from the one for the samples. AUFS, Absorbance Units Full Scale. 


\section{Discussion}

Currently, few scientific articles relate $\mathrm{KC}$ with dry eye syndrome [8]. Recent studies of the tears of KC patients have focused on the presence of pro-inflammatory molecules and their role on ectasia progression [11,34-36]. As we previously mentioned, the Dry Eye Workshop, published in 2007, includes the inflammation of ocular surface as one of the possible signs of dry eye syndrome [29]. This led us to consider that there could be a close relationship between dry eye syndrome and $\mathrm{KC}$. This study evaluated and compared the signs of $\mathrm{Ap}_{4} \mathrm{~A}$ and $\mathrm{Ap}_{5} \mathrm{~A}$ concentrations and another signs and symptoms of dry eye in patients with $\mathrm{KC}$ and the influence of wearing GP contact lenses.

Previous studies examining dry eye signs and symptoms in $\mathrm{KC}$ patients who wore RGP lenses, had shown that the KC patients had increased concentrations of $\mathrm{Ap}_{4} \mathrm{~A}$ in their tears [10,37]. Current results match better with the most recent study published [37] than the older manuscript [10]. This is due to the low tear volume found in the $\mathrm{KC}$ patient of the study published in 2014, being almost 4 times lower than the other two studies (matched with $\mathrm{Ap}_{4} \mathrm{~A}$ ratio between studies).

With the aim to evaluate if $\mathrm{KC}$ disease causes changes in dinucleotide concentration, the $\mathrm{KC}$ group was divided into RGP wearers and RGP non-wearers. And the findings are very significant, because non-RGP wearing $\mathrm{KC}$ patients showed 2 times higher $\mathrm{Ap}_{4} \mathrm{~A}$ concentration than healthy subjects without contact lenses $(0.417 \pm 0.313 \mu \mathrm{M}$ vs. $0.185 \pm 0.178 \mu \mathrm{M})[20]$. Therefore, $\mathrm{KC}$ may cause an increase in the concentration of $\mathrm{Ap}_{4} \mathrm{~A}$. Also, this study corroborates the effect of RGP on the concentration of $A_{4} \mathrm{~A}$ found in a previous study [22]. KC patients who wore RGP lenses had a 2 times higher Ap4A concentration than Kc patients who did not wear RGP lenses $(0.794 \pm 0.478 \mu \mathrm{M}$ vs. $0.417 \pm 0.313 \mu \mathrm{M})$.

The increase in $\mathrm{Ap}_{4} \mathrm{~A}$ in patients of $\mathrm{KC}$ may be due to a mechanical stimulation of the epithelial cells. It has been shown in both in vitro and in vivo studies, that the release of the nucleotides is driven by the shear stress produced on the corneal epithelial cells [23]. There are many studies that report a high prevalence of eye rubbing in patients with $\mathrm{KC}$, with percentages between $46 \%$ and $80 \%$ of eye rubbing $[3,6]$. It is therefore likely that friction between lids and cornea is a mechanical stimulus for the release of dinucleotides to the tear. Furthermore, contact lenses exert mechanical pressure on the corneal epithelium in each blink, which may also stimulate the release of dinucleotides. In some studies, in which tear samples were taken before and after contact lens wear, significantly increased concentrations of $\mathrm{Ap}_{4} \mathrm{~A}$ and $\mathrm{Ap}_{5} \mathrm{~A}$ were found $[22,38]$.

Nevertheless, KC patients who are not contact lens wearers also showed statistically higher concentrations of dinucleotides than the patients in the control group. The reasons for this observation are not clear, but we have several hypotheses. It might be possible that the increased curvature of the anterior surface of the cornea caused increased friction in each blink on the corneal surface which stimulates the release of the dinucleotides. No studies on the blinking frequency in $\mathrm{KC}$ patients has been performed but lower density of epithelial cells, primarily in the apical area have been reported, possibly due to the blinking stress [39,40]. Another possibility is the presence of dry eye associated with KC. There are some references in the literature that relate $\mathrm{KC}$ with dry eye but they describe the presence of cytokines such as interleukin-6 or Tumor necrosis factor (TNF- $\alpha$ ) and metalloprotease 9 (MMP-9) in the tears of patients with $\mathrm{KC}[12,41]$. These mediators are very important in the pathogenesis of dry eye $[42,43]$. In another study published in 2003, the authors reported a decrease in tear volume and TBUT in patients with $\mathrm{KC}$ [8].
Besides the concentration of dinucleotides, other signs and symptoms of dry eye in patients with $\mathrm{KC}$ were evaluated. When compared with the control group, we have found that patients with $\mathrm{KC}$ have less tear volume, reduced tear stability, and more corneal staining, all of which are statistically significant. These findings strongly suggest that the increased concentrations of dinucleotides in $\mathrm{KC}$ are mainly caused by the associated dry eye.

Regarding the tear volume and stability, $\mathrm{KC}$ patients who use contact lenses had significantly lower values than those who are nonusers. A possible reason could be that GP contact lenses disrupt normal tear physiology by thinning and breakup of the tear film lipid layer favoring the consequent increase in tear film evaporation [44]. Patients using contact lenses show a significant increase in the McMonnies test. These scores are considered marginal dry eye [45]. It seems possible that the use of rigid gas permeable contact lenses is related to dry eye symptoms in these $\mathrm{KC}$ patients, though it would be interesting to know how long each subjects had the disease as this may be an influential factor.

Dogru et al. [8] also indicated that $81.5 \%$ of their patients reported discomfort, irritation and foreign body sensation, although that study does not differentiate between contact lens wearers and non-wearers. Although, our KC patients are older, the percentage of patients diagnosed or treated of dry eye in the age range between 25 to 29 years old and 30-34 years old was similar [46] and moreover, signs and symptoms of dry eye worsen with age, but it is accepted that the age of onset is $45-50$ years old, which could be potentially linked to hormonal factors [47]. However, KC patients did not show a relationship between age and the parameters evaluated, which could be related to the narrow age range and low subject numbers in our study. A future study with a larger population may be necessary to evaluate the symptomatology of dry eye in KC patients as a function of age including ages up to 60-70 years old. Otherwise, the same number of patients was recruited from both places for both groups, control and $\mathrm{KC}$ group. We found no differences between places for any parameter evaluated, but the KC subgroups division in function of the centre gives us very small samples to make any conclusion. Therefore, more studies about environmental conditions and dry eye signs and symptoms in $\mathrm{KC}$ patients should be performed.

On the one hand, our patients using RGP lenses have no more staining than non-wearers. This would indicate that symptoms in these patients are not due to corneal damage but due to the contact lens wear. In the case of contact lens wearers, several types of contact lenses are currently fitted in $\mathrm{KC}$ patients, from soft contact lenses or GP corneal contact lenses to gas permeable scleral contact lenses or hybrid contact lenses $[48,49]$. On the other hand some studies have found that gas permeable contact lens wear increases the presence of pro-inflammatory molecules in tears of patients with KC [13]. Furthermore, several studies have demonstrated that soft contact lens wear provokes symptoms of dryness, especially at the end of the day [50], but no studies with scleral or hybrid contact lenses have been performed.

Finally, limitations of this study are the lack of patients with different severity of $\mathrm{KC}$. It might be interesting to investigate the influence of KC severity to confirm our lack of correlation between severity, dry eye signs and symptoms. Eye rubbing was reported by 13 keratoconus patients, 6 non-RPG lens wearers and 7 RGP wearers. The patients who related eye rubbing showed a higher concentration of dinucleotides and Mcmonnies score than non-rubbing eyes in both $\mathrm{KC}$ subgroups. However, the sample size for this parameter is too small for statistical analysis and more patients would be necessary to confirm this trend. 
In conclusion, the parameters evaluated in this study indicate that patients with $\mathrm{KC}$ have more severe signs and symptoms of dry eye than subjects without $\mathrm{KC}$, which could be exacerbated when the patient wears gas permeable contact lenses.

\section{Disclosure}

The authors do not have any financial interest on the materials and instruments used in this study.

\section{Acknowledgements}

This work was supported by grants from the Ministerio de Ciencia e Innovación (SAF2010-16024 and SAF2013-44416-R) and RETICS (RD12/0034/0003).

The authors want to thank Thomas Johnson and Vimala Punsammy for helping in the preparation of the manuscript.

\section{References}

[1] M. Romero-Jimenez, J. Santodomingo-Rubido, J.S. Wolffsohn, Keratoconus: a review, Cont. Lens Anterior Eye 33 (2010) 157-166.

[2] Y.S. Rabinowitz, Keratoconus, Surv. Ophthalmol. 42 (1998) 297-319.

[3] C.W. McMonnies, G.C. Boneham, Keratoconus, allergy, itch, eye-rubbing and hand-dominance, Clin. Exp. Optom. 86 (2003) 376-384

[4] M. Cristina Kenney, D.J. Brown, The cascade hypothesis of keratoconus, Cont. Lens Anterior Eye 26 (2003) 139-146.

[5] H.Y. Chang, J. Chodosh, The genetics of keratoconus, Semin. Ophthalmol. 28 (2013) 275-280.

[6] C.W. McMonnies, Mechanisms of rubbing-related corneal trauma in keratoconus, Cornea 28 (2009) 607-615.

[7] B. Yeniad, N. Alparslan, K. Akarcay, Eye rubbing as an apparent cause of recurrent keratoconus, Cornea 28 (2009) 477-479.

[8] M. Dogru, H. Karakaya, H. Ozcetin, H. Erturk, A. Yucel, A. Ozmen, et al., Tear function and ocular surface changes in keratoconus, Ophthalmology 110 (2003) $1110-1118$.

[9] K.J. Cho, J.W. Mok, M.Y. Choi, J.Y. Kim, C.K. Joo, Changes in corneal sensation and ocular surface in patients with asymmetrical keratoconus, Cornea 32 (2013) 205-210.

[10] G. Carracedo, A. Recchioni, N. Alejandre-Alba, A. Martin-Gil, A. Crooke, I.J. Morote, et al., Signs and symptoms of dry eye in keratoconus patients: a pilot study, Curr. Eye Res. (2014) 1-7.

[11] I. Lema, D. Brea, R. Rodriguez-Gonzalez, E. Diez-Feijoo, T. Sobrino, Proteomic analysis of the tear film in patients with keratoconus, Mol. Vis. 16 (2010) 2055-2061.

[12] I. Lema, T. Sobrino, J.A. Duran, D. Brea, E. Diez-Feijoo, Subclinical keratoconus and inflammatory molecules from tears, Br. J. Ophthalmol. 93 (2009) $820-824$.

[13] I. Lema, J.A. Duran, C. Ruiz, E. Diez-Feijoo, A. Acera, J. Merayo, Inflammatory response to contact lenses in patients with keratoconus compared with myopic subjects, Cornea 27 (2008) 758-763.

[14] A.J. Bron, M.D. Abelson, G. Ousler, E.I. Pearce, A. Tomlinson, N. Yokoi, et al., Methodologies to diagnose and monitor dry eye disease: report of the Diagnostic Methodology Subcommittee of the International Dry Eye WorkShop (2007), Ocul. Surf. 5 (2007) 108-152.

[15] J. Pintor, A. Peral, T. Pelaez, S. Martin, C.H. Hoyle, Presence of diadenosine polyphosphates in the aqueous humor: their effect on intraocular pressure, J. Pharmacol. Exp. Ther. 304 (2003) 342-348.

[16] J. Pintor, G. Carracedo, M.C. Alonso, A. Bautista, A. Peral, Presence of diadenosine polyphosphates in human tears, Pflugers Arch. 443 (2002) 432-436.

[17] J. Pintor, A. Peral, C.H. Hoyle, C. Redick, J. Douglass, I. Sims, et al., Effects of diadenosine polyphosphates on tear secretion in New Zealand white rabbits, J. Pharmacol. Exp. Ther. 300 (2002) 291-297.

[18] A. Peral, P. Loma, B. Yerxa, J. Pintor, Topical application of nucleotides increase lysozyme levels in tears, Clin. Ophthalmol. 2 (2008) 261-267.

[19] J. Pintor, A. Bautista, G. Carracedo, A. Peral, UTP and diadenosine tetraphosphate accelerate wound healing in the rabbit cornea, Ophthalmic Physiol. Opt. 24 (2004) 186-193.

[20] A. Peral, G. Carracedo, M.C. Acosta, J. Gallar, J. Pintor, Increased levels of diadenosine polyphosphates in dry eye, Invest. Ophthalmol. Vis. Sci. 47 (2006) 4053-4058.

[21] G. Carracedo, A. Peral, J. Pintor, Diadenosine polyphosphates in tears of Sjogren syndrome patients, Invest. Ophthalmol. Vis. Sci. 51 (2010) 5452-5459.
[22] G. Carracedo, J.M. Gonzalez-Meijome, J. Pintor, Changes in diadenosine polyphosphates during alignment-fit and orthokeratology rigid gas permeable lens wear, Invest. Ophthalmol. Vis. Sci. 53 (2012) 4426-4432.

[23] G. Carracedo, A. Guzman-Aranguez, P. Loma, J. Pintor, Diadenosine polyphosphates release by human corneal epithelium, Exp. Eye Res. 113 (2013) $156-161$.

[24] K. Zadnik, J.T. Barr, T.B. Edrington, J.J. Nichols, B.S. Wilson, K. Siegmund, et al., Corneal scarring and vision in keratoconus: a baseline report from the Collaborative Longitudinal Evaluation of Keratoconus (CLEK) Study, Cornea 19 (2000) 804-812.

[25] J.R. Williams, The declaration of Helsinki and public health, Bull. World Health Organ. 86 (2008) 650-652.

[26] C.G. Begley, B. Caffery, R.L. Chalmers, G.L. Mitchell, Dry Eye Investigation Study Group, Use of the dry eye questionnaire to measure symptoms of ocular irritation in patients with aqueous tear deficient dry eye, Cornea 21 (2002) 664-670.

[27] R.L. Chalmers, C.G. Begley, B. Caffery, Validation of the 5-Item Dry Eye Questionnaire (DEQ-5): discrimination across self-assessed severity and aqueous tear deficient dry eye diagnoses, Cont. Lens Anterior Eye 33 (2010) 55-60.

[28] O.P. van Bijsterveld, Diagnostic tests in the Sicca syndrome, Arch. Ophthalmol. 82 (1969) 10-14

[29] The definition and classification of dry eye disease: report of the Definition and Classification Subcommittee of the International Dry Eye WorkShop, The ocular surface (2007) 5 75-92

[30] R.L. Terry, C.M. Schnider, B.A. Holden, R. Cornish, T. Grant, D. Sweeney, et al., CCLRU standards for success of daily and extended wear contact lenses, Optom. Vis. Sci. 70 (1993) 234-243.

[31] J. Pintor, M. Torres, M.T. Miras-Portugal, Carbachol induced release of diadenosine polyphosphates -, Life Sci. 48 (1991) 2317-2324.

[32] J. Pintor, M.A. Diaz-Rey, M. Torres, M.T. Miras-Portugal, Presence of diadenosine polyphosphates - Ap4A and Ap5 A - in rat brain synaptic terminals $\mathrm{Ca}^{2+}$ dependent release evoked by 4-aminopyridine and veratridine, Neurosci. Lett. 136 (1992) 141-144.

[33] T.T. McMahon, L. Szczotka-Flynn, J.T. Barr, R.J. Anderson, M.E. Slaughter, J.H. Lass, et al., A new method for grading the severity of keratoconus: the Keratoconus Severity Score (KSS), Cornea 25 (2006) 794-800.

[34] S.A. Balasubramanian, D.C. Pye, M.D. Willcox, Effects of eye rubbing on the levels of protease, protease activity and cytokines in tears: relevance in keratoconus, Clin. Exp. Optom. 96 (2013) 214-218.

[35] S.A. Balasubramanian, S. Mohan, D.C. Pye, M.D. Willcox, Proteases, proteolysis and inflammatory molecules in the tears of people with keratoconus, Acta Ophthalmol. (Copenh.) 90 (2012) e303-9.

[36] M. Fodor, B.L. Kolozsvari, G. Petrovski, B.A. Kettesy, P. Gogolak, E. Rajnavolgyi, et al., Effect of contact lens wear on the release of tear mediators in keratoconus, Eye \& contact lens 39 (2013) 147-152.

[37] G. Carracedo, M.S. Blanco, A. Martin-Gil, W. Zicheng, J.C. Alvarez, J. Pintor Short-term effect of scleral lens on the dry eye biomarkers in keratoconus, Optom. Vis. Sci. 93 (2016) 150-157.

[38] A. Guzman-Aranguez, A. Crooke, A. Peral, C.H. Hoyle, J. Pintor, Dinucleoside polyphosphates in the eye: from physiology to therapeutics, Prog. Retin. Eye Res. 26 (2007) 674-687.

[39] K.H. Weed, C.J. MacEwen, A. Cox, C.N. McGhee, Quantitative analysis of corneal microstructure in keratoconus utilising in vivo confocal microscopy, Eye 21 (2007) 614-623.

[40] R.L. Niederer, D. Perumal, T. Sherwin, C.N. McGhee, Laser scanning in vivo confocal microscopy reveals reduced innervation and reduction in cell density in all layers of the keratoconic cornea, Invest. Ophthalmol. Vis. Sci. 49 (2008) 2964-2970.

[41] I. Lema, J.A. Duran, Inflammatory molecules in the tears of patients with keratoconus, Ophthalmology 112 (2005) 654-659.

[42] C.S. De Paiva, R.M. Corrales, A.L. Villarreal, W.J. Farley, D.Q. Li, M.E. Stern, et al., Corticosteroid and doxycycline suppress MMP-9 and inflammatory cytokine expression, MAPK activation in the corneal epithelium in experimental dry eye, Exp. Eye Res. 83 (2006) 526-535.

[43] L. Luo, D.Q. Li, R.M. Corrales, S.C. Pflugfelder, Hyperosmolar saline is a proinflammatory stress on the mouse ocular surface, Eye Contact Lens 31 (2005) 186-193.

[44] L.C. Thai, A. Tomlinson, M.G. Doane, Effect of contact lens materials on tear physiology, Optom. Vis. Sci. 81 (2004) 194-204.

[45] K.K. Nichols, J.J. Nichols, G.L. Mitchell, The reliability and validity of McMonnies dry eye index, Cornea 23 (2004) 365-371.

[46] C. Yazdani, T. McLaughlin, J.E. Smeeding, J. Walt, Prevalence of treated dry eye disease in a managed care population, Clin. Ther. 23 (2001) 1672-1682.

[47] M.A. Lemp, Advances in understanding and managing dry eye disease, Am. J. Ophthalmol. 146 (2008) 350-356.

[48] G. Carracedo, J.M. Gonzalez-Meijome, D. Lopes-Ferreira, J. Carballo, L. Batres, Clinical performance of a new hybrid contact lens for keratoconus, Eye Cont. Lens 40 (2014) 2-6. 
[49] E. van der Worp, D. Bornman, D.L. Ferreira, M. Faria-Ribeiro, N. Garcia-Porta, J.M. Gonzalez-Meijome, Modern scleral contact lenses: a review, Cont. Lens Anterior Eye (2014).
[50] J.M. Gonzalez-Meijome, M.A. Parafita, E. Yebra-Pimentel, J.B. Almeida, Symptoms in a population of contact lens and noncontact lens wearers under different environmental conditions, Optom. Vis. Sci. 84 (2007) 296-302. 